
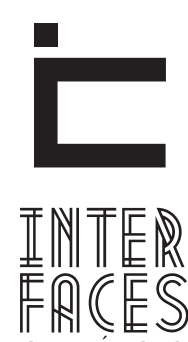

CIENTIIFICAS

HUMANAS E SOCIAIS

ISSN IMPRESSO 2316-3348

E-ISSN 2316-3801

DOI - 10.17564/2316-3801.2018v7n1p45-58

\title{
REVISÃo SISTEMÁTICA: INTERSECÇ̃̃o ENTRE AS TEORIAS DE ANÁLISE DE REDE, DESENVOLVIMENTO REGIONAL E GEOGRAFIA ECONÔMICA
}

\author{
SYSTEMATIC REVIEW: THE INTERSECTION BETWEEN THE THEORIES OF NETWORK ANALYSIS, \\ REGIONAL DEVELOPMENT AND ECONOMIC GEOGRAPHY
}

REVISIÓN SISTEMÁTICA: INTERSECCIÓN ENTRE LAS TEORÍAS DE ANÁLISIS DE RED, DESARROLLO REGIONAL Y GEOGRAFÍA ECONÓMICA

Bárbara Ádria Oliveira Farias Fernandes ${ }^{1}$ José Luiz Nunes Fernandes ${ }^{3}$
Cyntia Meireles Martins ${ }^{2}$

relacionados ao tema. Como conclusão identificou-se que as áreas do conhecimento pesquisadas, possuem intersecções teóricas em relação aos seguintes macros temas: fomento à competitividade; relacionamento entre atores e visão de atuação em espaços geográficos.

\section{PALAVRAS-CHAVE}

Competitividade. Relação entre Atores. Espaços Geográficos. 


\section{ABSTRACT}

The 1980s was prodigal regarding the studies of the network analyses, the inter-company relations and the connection with the economic geography. In this sense, with the source of data and literature on the topic, and by means of a systematic review, the objective of this work is to situate the concepts of network analysis, regional development and economic geography, and finally, the theoretical concepts starting from the literature review. Thus, it was researched if these theories are crossed or if the intersection occurs, organizing the theoretical foundations from the

\section{RESUMEN}

La década de 1980 fue pródiga en cuanto a los estudios de análisis de redes, las relaciones interempresariales y la conexión con la geografía económica. En este sentido, con fuente de datos y literatura sobre el tema y por medio de revisión sistemática, el trabajo tuvo como objetivo situar los conceptos de análisis de red, desarrollo regional y geografía económica y, por último, teóricos a partir de la revisión de la literatura. Con ello, se investigó si esas teorías se entrecortan u ocurre intersección, organizándose los fundamentos teóricos a partir de la revisión de la literatura en trabajos relacionados review of literature in the studies related to the topic. As a conclusion, it was identified that the surveyed knowledge areas have theoretical intersections in relation to the following macro topics: the promotion of the competitiveness; the relationship between actors and the vision of acting in geographical areas.

\section{KEYWORDS}

Competitiveness. Relationship Between Actors. Geographical Areas

al tema. Como conclusión se identificó que las áreas del conocimiento investigadas poseen intersecciones teóricas en relación a los siguientes macro temas: el fomento a la competitividad; la relación entre actores y la visión de actuación en espacios geográficos.

\section{PALABRAS CLAVE}

Competitividad. Relación entre actores. Espacios geográficos 


\section{INTRODUCÇÃO}

Buscar evidências de pesquisa para guiar estudos empíricos é uma das razões para elaboração de estudos que sintetizem a literatura, mas não é a única. Estudos pautados em revisões sistemáticas são métodos explícitos que permitem reproduções. Esse tipo de estudo possibilita o desenvolvimento de projetos e aponta novos rumos para futuras investigações.

Diante disto, a década de 1980 foi pródiga quanto aos estudos de análises de redes, as relações interempresariais e a conexão com a geografia econômica (AMIN; THRIFT, 1994). Observa-se, por outro lado, que a geografia econômica é uma área do conhecimento interdisciplinar e eclética que subjaze diversidade de disciplinas correlacionadas como a própria economia, estudos organizacionais, sociologia entre outras.

Quanto a relação sinérgica da geografia econômica com os principais temas da presente pesquisa, Chung-Yeung (2014) entende que a geografia econômica possui interface com as relações interorganizacionais e o desenvolvimento regional.

Ainda em relação ao desenvolvimento regional, autores como Bathelt e Glückler (2003) e Yeung (2005) desenvolveram o conceito de bens relacionais para analisar o papel das relações institucionais e sociais com o objetivo de fortalecer e consolidar a teoria voltada ao desenvolvimento local e regional.

Necessário é o entendimento do impacto da economia em escala global sobre a economia regional. Diante desse desafio, Pitteri, Saes e Bresciani (2015) posicionam-se no sentido de que o crescente interesse nos efeitos da economia em escala global sobre as regiões confirma a importância da melhor compreensão de como as forças locais se articulam em torno de diferentes concepções para o desenvolvimento local sustentável e de longo prazo.

Justifica-se, portanto a importância desse estudo que busca contribuir com a comunidade científica em promover o avanço do conhecimento e, de forma especial, por meio do entendimento da intersecção entre as teorias subjacentes a análise de redes, desenvolvimento regional e a geografia econômica.
Estudos desenvolvidos (MALMBERG; MASKELL, 1997; MASKELL; MALMBERG, 1999; PHELPS, 2004) demonstram que a aglomeração espacial e a formação de redes de produção territorial são temas relevantes e negligenciados na análise de redes, demandando, portanto, estudos e pesquisas.

Diante do contexto antes descrito emerge a seguinte questão de pesquisa: Como ocorre - e se ocorre - $\mathrm{a}$ intersecção entre as teorias de análise de rede, desenvolvimento regional e geografia econômica?

Nesse sentido o objetivo do artigo é situar os conceitos de análise de rede, desenvolvimento regional e geografia econômica, organizando os fundamentos teóricos a partir de revisão da literatura em trabalhos relacionados ao tema e pesquisar se essas teorias se entrecortam ou ocorre intersecção.

\section{REVISÃO DA LITERATURA}

\subsection{TEORIA DE ANÁLISE DE REDES}

Entende-se rede como atores interconectados e nessa direção Balestrin e Vargas (2004) explicam que, no campo de estudos das Ciências Sociais Aplicadas, o termo rede designa um conjunto de pessoas ou organizações interligadas direta ou indiretamente.

Com visão gerencial, Bowersox (1990) expressa o entendimento que uma rede é reflexo do desejo entre os participantes de eliminar as duplicidades de atividades nas interfaces da cadeia de valor agregado e de reduzir desperdícios de recursos. São arranjos de longo prazo criados entre empresas diferentes com um propósito em comum: o de criar e manter vantagem competitiva frente aos concorrentes fora da rede, minimizando seus custos de atividades e os custos de coordenação (ROHDEN et al., 2012). Avança-se para Green (1993) ao posicionar-se no sentido de que o ponto forte da abordagem de rede é que trata o fenômeno como todo e, assim, permite a descoberta de relações invisíveis para a análise de partes distintas.

Pode-se dizer que para entender as Redes de Negócios, uma boa estratégia é compreendê-las, de forma sis- 
temática, por meio das conceituações de nós e elos. Nós são os pontos da rede, ou seja, os agentes constituintes dela, enquanto os elos são as ligações entre esses nós (CUNHA; PASSADOR; PASSADOR, 2011).

Explica-se também a atuação em rede de negócios por meio da dependência de recursos, fato que motiva as organizações a procurar relações com outras organizações (ALDRICH; FELDMAN, 1996; PFEFFER; NOWAK, 1976).

Para Chung Yeung (2014), as redes empresariais, por exemplo, estão frequentemente embasadas em laços interpessoais, em fluxos de informações informais, no compartilhamento dos recursos, no aprendizado e no conhecimento descentralizado.

As parcerias tornam-se equilibradas quando os custos são mensurados e o conhecimento sobre a lucratividade de produtos, de segmento de clientes e visão holística para negociação entre os parceiros da cadeia de negócios são estimulados (GUERREIRO et al., 2011).

Nesse sentido, Porter (1992) complementa, afirmando que a empresa ganha vantagem competitiva quando executa suas atividades estratégicas com custos menores e executadas com mais eficiência que a concorrente. Portanto, as empresas também se reúnem em redes de relações in loco em virtude de competirem e cooperarem entre si simultaneamente em plataformas locais e transnacionais (CHUNG YEUNG, 2014, p. 430).

Diante desta realidade aflora o termo coopetição, assim, autores como Nalebuff e Brandenburger (1996); Zineldin (2004); Luo (2005); bem como Hollerweger e Rohden (2012) entendem que nos relacionamentos coopetitivos as empresas podem cooperar e competir simultaneamente. Portanto, competir e cooperar são ações presentes nas redes de negócios.

Para Fleury e Fleury (2010), a busca por diferenciação no mercado torna-se cada vez mais acirrada, devido a fenômenos como, por exemplo, a existência do mercado globalizado, o constante avanço das tecnologias e o fácil acesso às informações.

Observa-se que parte significante dos estudos (GIL et al., 2012; JUNQUEIRA FILHO, 2006; OLIVEIRA, 2014) está voltada para o desenvolvimento regional e, de forma especial, alicerçados no conceito de "bens relacionais”, o qual, tenta explicar as relações insti- tucionais e sociais e suas contribuições para o desenvolvimento local e regional.

\subsection{DESENVOLVIMENTO REGIONAL}

Nos entendimentos de Gil, Oliva e Silva (2012), a globalização não trouxe para os menos abastados da Terra o que prometeu, por outro lado, contribuiu sobremaneira para o enfraquecimento do papel dos Estados nacionais, com a consequente limitação de seu poder de formulação e execução de políticas sociais e econômicas. Desse modo, uma das reações mais evidentes é constituída pela valorização do regional como contraponto ao global.

Essa tendência é ratificada pelas palavras de Junqueira Filho (2006, p. 15):

A regionalidade se apresenta como potencializadora dos processos globalizantes, sendo contraponto as consequências da globalização por permitir, além da sobrevivência, o desenvolvimento empresarial distinto, descontínuo, com dinâmica própria reorganizando elementos já existentes objetivando a criação de novas possibilidades, as quais, muitas vezes desenvolvidas "à sombra do estado".

Oliveira (2014), ao abordar sobre o tema regionalidade, traz a afirmação de que a união de atores sociais em torno das necessidades de uma região é reconhecida como novo campo do conhecimento por conta da necessidade de reação ante a globalização e, ao mesmo tempo, como consequência dela, verifica-se em praticamente toda parte do globo o aparecimento de movimentos e organizações que preconizam a valorização do regional e do local.

Para Gil, Garcia e Klink (2012, p. 18), o conceito de regionalidade é:

Qualidade de ser uma região, conjunto de propriedades e circunstâncias que distinguem um espaço e que permitem sua comparação com outras regiões. Consciência coletiva que une os habitantes de uma determinada região em torno de sua cultura, sentimentos e problemas; formação social que surge da articulação de esforços conjuntos das autoridades públicas, dos 
empresários, dos representantes de segmentos da sociedade civil e dos representantes de outras organizações, no espaço da região que pode ser geográfico, administrativo, econômico, político, social e cultural.

0 conceito de regionalidade, num primeiro momento, pode confundir-se com o de regionalismo. Porém, regionalidade se vincula à reorganização do Estado local a partir de novas formas de parcerias que emergem para guiar e promover o desenvolvimento de recursos locais. Assim, só se pode falar em regionalidade quando se verificar a efetiva cooperação, entre as instâncias de governo regional e os vários segmentos da sociedade civil, com o propósito de promover o desenvolvimento (OLIVEIRA, 2014).

Dentro dessa visão, cita-se Zambanini (2014) que, ao falar de inovação e desenvolvimento local, entende que esses trazem à reflexão para que o território possua a inovação como estratégia fundamental do seu desenvolvimento. Para Gil (et al, 2012b) território é o espaço geográfico onde os atores habitam e exercem suas atividades, portanto, possuem vínculos, desse modo, torna-se necessário que essa consciência seja culturalmente construída e não imposta. Com o mesmo entendimento Gil (et al., 1999; MENDES; BELLINI, 2011 e 2012a) expressam que são necessários vários anos para que a região possa construir essa cultura de inovação, configurando-se como processo de médio e longo prazos em que num primeiro momento os resultados podem parecer pouco concretos.

Essa nova concepção deveria passar por um processo de aprendizado dos diversos atores, os quais deveriam estar dispostos ao diálogo sobre seus interesses, mas com o ideal voltado para um consenso das diferenças o que não é fácil conseguir a unanimidade na postura entendida como ideal (MENDES; BELLINI, 2011).

Por outro lado e, com a visão de território-Estado na intenção de caracterizar a regionalidade, Gil, Garcia e Klink (1999) afirmam que a convivência de grupamentos, com forças políticas e econômicas em busca de um objetivo comum, resulta no surgi- mento do conceito de regionalidade, que se caracteriza, portanto, como a reorganização do Estado local com novas formas de parcerias que emergem para guiar e promover o desenvolvimento local, entre as instâncias de governo regional e os vários segmentos da sociedade civil, isto com o propósito de promover o desenvolvimento regional. Storper (1997) também acredita que o desenvolvimento territorial seja incorporado nas redes de bens relacionais e na proximidade espacial, especialmente em escalas locais e regionais.

Diante desse contexto, discute-se os benefícios sociais e econômicos advindos da globalização dos mercados quando relacionados aos espaços regionais/locais, assim, o que alicerça a discussão está situado no debate contemporâneo intelectual e político sobre a globalização e suas diversas implicações com o desenvolvimento local e regional (COX, 1997; YEUNG, 2005; KELLY, 1999; OLDS et al., 1999; PECK; YEUNG, 2005; DICKEN, 2004).

Por outro lado, e, ao incluir os desafios organizacionais na discussão globalização e regionalidade, admite-se que a espessura institucional possui desafios quando envolve as variáveis empresas e regiões. Nesta direção, Chung Yeung (2014) afirma que a espessura institucional é uma faca de dois gumes, na medida em que o crescimento das empresas e o desenvolvimento regional estão envolvidos. Scott (1988), tivera pensamento semelhante ao de Chung Yeung (2014), ao entender que a espessura institucional pode ser um obstáculo para desenvolver e fazer crescer atitudes pelo fato de que hábitos teimosamente disfuncionais são solidamente encrustados no sistema econômico local.

Diante disto, entende-se que as aglomerações territoriais são elementos que geram vantagens com as interdependências, como também diminui riscos, possibilita maior flexibilidade, minimização de custos de transação e aumento da especialização (GIL; OLIVA; SILVA, 2012; GIL; GARCIA; KLINK, 2012). Em caso extremo, sem proximidade geográfica, estas vantagens não existiriam (PITTERI; SAES; BRESCIANI, 2015). 


\subsection{GEOGRAFIA ECONÔMICA}

A geografia econômica é uma área do conhecimento eclética que delineia uma gama de disciplinas cognatas, como a economia, a sociologia, os estudos organizacionais e a política econômica e desde a metade da década de 1980, as redes e as inserções vêm dominando o léxico na sociologia econômica, nos estudos organizacionais e no gerenciamento estratégico (CHUNG YEUNG, 2014).

Martim (1996) explica que durante a década de 1980 os estudos voltados a Geografia Econômica possuíam dos eixos: (i) dinâmica de localização industrial; (ii) processo de desenvolvimento regional desigual. Esses eixos eram derivados dos conceitos e teorias da economia neoclássica de Keynes e Marx. Destaca-se também os entendimentos de Peck (2005) e Grabher (2006), quando expressam que, apesar do envolvimento teórico sobre redes e a imersão, possuir profundo impacto sobre a sociologia econômica e estudos organizacionais, sua difusão na área voltada à geografia econômica permaneceu relativamente incipiente até o início dos anos de 1990.

Por outro lado, autores (CLARK et al., 2000; SHEPPARD; BARNES, 2000; BARNES et al., 2003) afirmam que a geografia econômica é uma área do conhecimento humano fundamentalmente interessada em descrever e explicar a organização espacial da atividade econômica. Essa especialidade da economia pode ser analisada em relação à localização no espaço, no local e na escala dos processos econômicos, além dos resultados direcionados aos atores locais (CHUNG YEUNG, 2014).

Evidencia-se o entendimento de Domingues (2015) quando expressa que diante das novas realidades econômica nenhuma das principais escolas de economia, explica os acontecimentos e mudanças das últimas décadas. Daí a necessidade de repensar as teorias, premissas e os princípios da moderna geografia econômica. Chung Yeung (2014) avança quando expressa que a concepção dos atores e de suas relações de poder aprimora o trabalho dos geógrafos econômicos sobre os sistemas industriais ao focalizar principalmente as conexões econômicas entre empresas.
Identifica-se no estudo de Allen (2003) que as relações entre os espaços geográficos e o poder estão entrelaçados com a espacialidade, portanto, espaço geográfico econômico e poder são inter-relacionados. Deste modo, o que instiga é saber exatamente qual o conceito de "poder" no contexto da geografia econômica. Nesse pensamento, Lukes (1996, p. 84) explica que, nas ciências sociais, o poder é um conceito especialmente difícil e desafiador e expressa seu entendimento:

\begin{abstract}
Existem várias respostas, todas profundamente próximas, que respondem aos nossos interesses tanto sobre os resultados quanto sobre as locações do poder. Talvez isso explique por que, em nossos julgamentos irrefletidos e comparações de poder, normalmente entendemos o que queremos dizer e temos pouca dificuldade de entender um ao outro, ainda que cada tentativa para uma resposta única à questão tenha falhado e pareça fadada ao fracasso.
\end{abstract}

Allen (2003) continua e diz que o poder tem um efeito relacional nas interações sociais, portanto, define poder como os efeitos relacionais da capacidade de influência e o exercício dessa capacidade por meio de prática de atores específicos.

Entende-se que as relações entre organizações é o locus do poder, Chung Yeung (2014) vê o poder como os efeitos emergentes da prática social entre atores em diferentes organizações e, na visão dessa relação, entende o poder como um atributo emergente, de maneira que a soma das relações heterogêneas é muito maior do que das partes individuais.

Neste panorama de estudo emerge a perspectiva de Redes de Produção Global sob o acrônimo de RPG. Chung Yeung (2014) explica que a RPG se fundamenta na literatura da geografia econômica, foca as relações entre organizações e explica o poder emergente nas redes relacionais.

É possível entender a RPG com visão espacial regional macro, ou seja, com visão global. Dicken (2004) explica que finalmente o quadro de cadeia de valor encontrará o caminho na geografia econômica, fato que permite desenvolver estudos sobre as organizações transnacionais e o desenvolvimento regional. 


\subsubsection{A NOVA ECONOMIA INSTITUCIONAL [NEI] E A GEOGRAFIA ECONÔMICA}

Coase (1937), ao apresentar o trabalho The Nature of Firme já referenciara o papel das instituições no cenário econômico. Pitteri, Saes e Bresciani (2015) explicam que a Nova Economia Institucional (NEI) aborda o papel das instituições sob dois níveis analíticos distintos: (i) o ambiente institucional e, (ii) as estruturas de governança, que contemplam tanto as macroinstituições, estabelecendo as bases para as interações entre os seres humanos, quanto as microinstituições, que regulam uma transação específica.

Destaca-se o entendimento de Meheir (1995), quando expressa que, a partir das instituições, os sistemas econômicos podem ser estudados. Para Domingues (2015), a NEl consolidou-se na década de 1990 após quarenta anos de estudos e pesquisas alicerçadas em conceitos e métodos de análises econômicas, cita-se dentre os diversos pesquisadores da NEI: Coase (1937), Williamson (1993) e North (1993). Ao ampliar a velha economia institucionalista Bazzoli e Dutraive (1995) focam no centro da teoria econômica a análise de processos empresariais por meio da ação coletiva com o intuito de melhor explicar as atividades econômicas e o capitalismo.

Domingues (2015) entende que a NEI considera o indivíduo como ponto de partida de mudança, mas dá importância às instituições, inclusive destacando como fundamentais as normas formais e informais, regras, leis e hábitos de conduta. É, portanto, uma ampliação de seus postulados, que levaria a entender melhor o comportamento dos agentes econômicos e políticos.

Para North (1993), as instituições são passíveis de mudanças e elas ocorrem de forma diferente. As instituições formais mudam em menor espaço de tempo e precisam da ação coerciva do Estado para serem observadas, enquanto as instituições informais não necessitam de ação coerciva do Estado, pois dependem das crenças de seus seguidores - a sociedade.

Kalmanovitz (2003) expressa que as crenças não mudam em espaços de tempo curtos, ao contrário, demoram muito para se consolidar, deste modo admite que a chave do crescimento e desenvolvimento econô- mico e social estável e sustentável, não está, apenas, na manipulação de variáveis macroeconômicas, e sim, nas relações entre indivíduos em sua atividade cotidiana, no interior das empresas e no aparato do Estado.

Análise efetuada por Williamson (1993) atribuiu outra perspectiva para as instituições formais ao segmentá-la em dois níveis: (i) as regras que atuam em nível macro, como as leis que regulam um país e; (ii) as regras que atuam em nível micro, como os contratos entre sócios de uma empresa. Essas perspectivas ratificam a pluralidade intrínseca da definição de instituições.

Atribui-se a Coase, pioneiro no estudo da Teoria dos Custos de Transação (TCT) a inciativa da análise institucional, pois a TCT enfatiza que os custos de transação tendem a aumentar quando as entidades são consideradas frágeis. Em tal princípio, Williamson avança ao mostrar que no contexto, em que o mercado é insuficiente, a instituição impõe-se ao coordenar as atividades (DOMINGUES, 2015). Análise de North (1993) diz que a TCT no processo do desenvolvimento econômico pode ser instrumento pertinente, tanto para a história econômica quanto para o estudo da dinâmica institucional.

\section{PROCEDIMENTOS METODOLÓGICOS}

Entende-se que a revisão sistemática, assim como outros tipos de estudo de revisão, é uma forma de pesquisa que utiliza como fonte de dados a literatura sobre determinados temas (SAMPAIO; MANCINI, 2007). Portanto, à aplicação desta revisão no presente artigo dar-se ao situar os conceitos de análise de rede, desenvolvimento regional e geografia econômica e organizar os fundamentos teóricos a partir de revisão da literatura.

Optou-se pela revisão sistemática por entender que essa permite adicionar um maior entendimento de resultados relevantes ao invés de limitar conclusões a leitura de alguns artigos, além de possibilitar a avaliação da consistência e generalização dos resultados individualizados dos autores.

Buscou-se inicialmente levantar autores clássicos e contemporâneos, quantos aos temas que permeiam a pesquisa, seus posicionamentos e entendimentos; 
em seguida, e por meio de revisão sistemática, procurou-se identificar onde os posicionamentos causam intersecção entre si, de outra maneira, onde existe concordância ou similaridade nos entendimentos das teorias alvo da pesquisa.
Em seguida, as teorias que sustentam a pesquisa serão alvo de triangulação. Com tal intenção, Patton (1987) explica que, dentre os tipos de triangulação, a de teorias permite identificar as identidades entre elas.

Figura 1 - Triangulação das Teorias

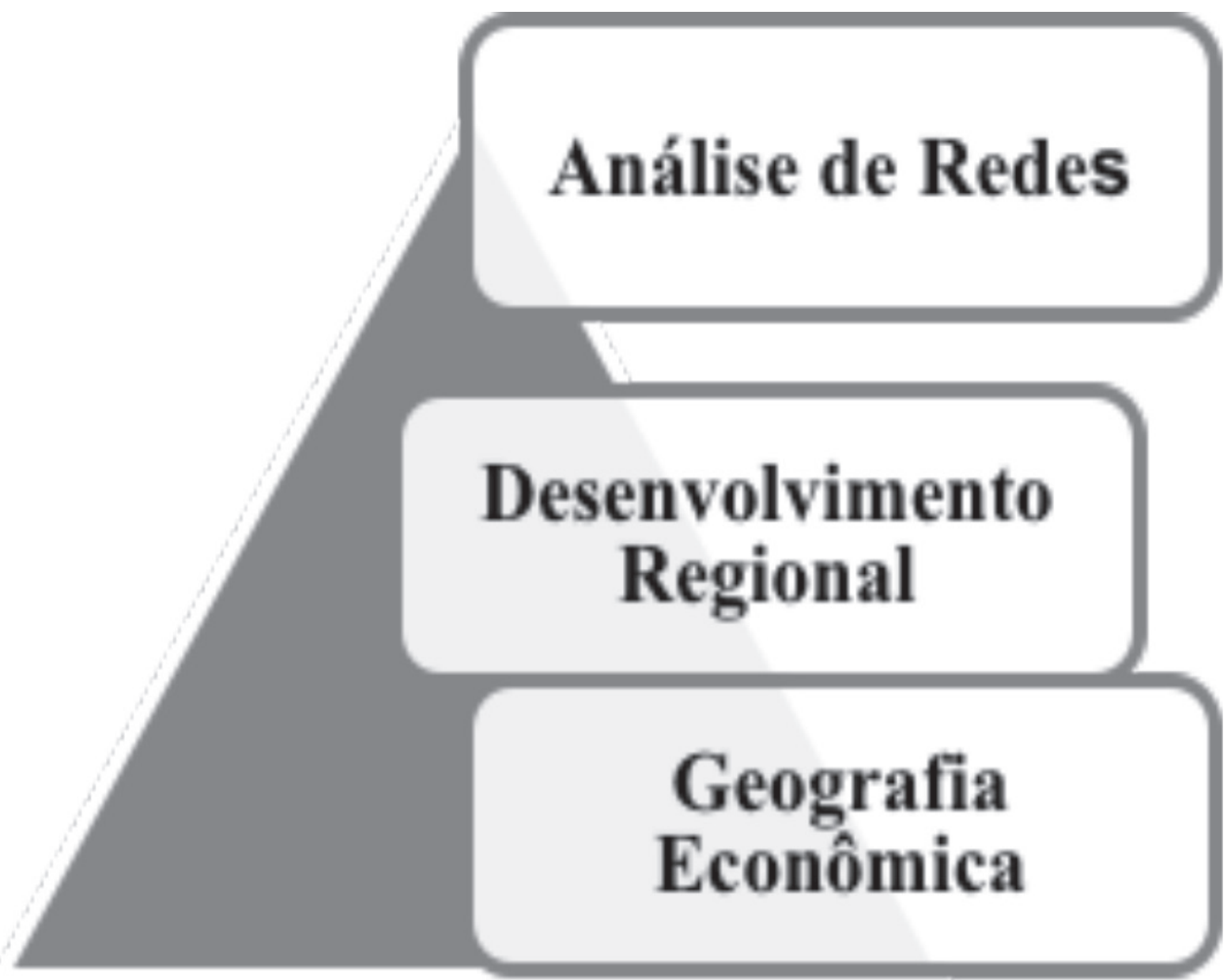

Fonte: Elaborado pelos autores (2016). 


\section{RESULTADOS}

Com o objetivo de buscar identificar existência de intersecção entre as teorias estudas, resume-se o Quadro 1.

Quadro 1 - Intersecção entre as teorias de Redes, Desenvolvimento Regional e Geografia Econômica

\begin{tabular}{|c|c|c|}
\hline TEORIAS & INTERSECÇÃO & AUTORES \\
\hline $\begin{array}{l}\text { 1.Ciências Sociais } \\
\text { 1.1Redes } \\
\text { 1.2Desenvolvimento Regional } \\
\text { 1.3Geografia Econômica }\end{array}$ & $\begin{array}{l}\text { Conjunto de pessoas } \\
\text { interligadas } \\
\text { Grupo de } \\
\text { indivíduos de uma região } \\
\text { Efeito relacional das interações sociais }\end{array}$ & $\begin{array}{l}\text { Balestrin; Vargas 2004; Bowersox, } \\
1990 . \\
\text { Bandeira, 1999; Oliveira, } 2011 . \\
\text { Allen, 2003; Chung Yeung,2014 }\end{array}$ \\
\hline $\begin{array}{l}\text { 2. Competitividade } \\
\text { 2.1 Redes } \\
\text { 2.2Desenvolvimento Regional } \\
2.3 \text { Geografia Econômica }\end{array}$ & $\begin{array}{l}\text { Criar e manter vantagem competitiva } \\
\text { Permite inovação estratégica } \\
\text { Cadeia de valor fomenta a } \\
\text { competitividade }\end{array}$ & $\begin{array}{l}\text { Rohden; Hollerweger; Ossani, 2012; } \\
\text { Porter, } 1992 . \\
\text { Zambanini 2014; Mendes; Bellini, } \\
2011 . \\
\text { Dicken,1986; Allen, } 2003\end{array}$ \\
\hline $\begin{array}{l}\text { 3. Relacionamento entre atores } \\
\text { 3.1 Redes } \\
\text { 3.2Desenvolvimento Regional } \\
\text { 3.3 Geografia Econômica }\end{array}$ & $\begin{array}{l}\text { Pessoas ou organizações interligadas } \\
\text { Capacidade de interação dos agentes } \\
\text { regionais } \\
\text { Efeito relacional das interações sociais }\end{array}$ & $\begin{array}{l}\text { Balestrin; } \\
\text { Vargas 2004;Rohden; Hollerweger; } \\
\text { Ossani, 2012. } \\
\text { Oliveira, } \\
\text { 2011; Mendes; Bellini, } 2011 . \\
\text { Dicken,1986; Allen, 2003; }\end{array}$ \\
\hline $\begin{array}{l}\text { 4. Visão de espaço geográfico } \\
\text { 4.1 Redes } \\
\text { 4.2Desenvolvimento Regional } \\
\text { 4.3 Geografia Econômica }\end{array}$ & $\begin{array}{l}\text { Plataforma locais e transnacionais } \\
\text { Grupo de indivíduos de uma região } \\
\text { Explicar a organização espacial da } \\
\text { atividade econômica }\end{array}$ & $\begin{array}{l}\text { Chung Yeung, } 2014 . \\
\text { Bandeira, 1999; Oliveira, } 2011 . \\
\text { Clark et al., 2000; Sheppard; Bar- } \\
\text { nes, 2000; Barnes et al., } 2003 .\end{array}$ \\
\hline
\end{tabular}

Fonte: Elaborado pelos autores (2016). 
O Quadro 2 demonstra que as teorias estudadas possuem intersecção nos seguintes aspectos:

Ciências Sociais: As teorias, em seus objetos de estudos, envolvem as pessoas físicas comuns e as entidades econômicas e o desafio de suas interações;

Competitividade: Reconhecem que os aglomerados empresariais fomentam a competitividade dos atores;

Relacionamento entre atores: Que as relações e trocas entre atores estão presentes em todas as três teorias estudadas;

Visão de espaço geográfico: As teorias identificam-se no sentido de reconhecer a atuação regional.

Ante ao que foi sintetizado no Quadro 1, é possível entender que existe consenso entre autores (BALESTRIN; VARGAS, 2004; BOWERSOX, 1990; BANDEIRA, 1999; OLIVEIRA, 2011; ALLEN, 2003; CHUNG YEUNG,2014) que as teorias de Redes, Desenvolvimento Regional e Geografia Econômica fazem parte da área do conhecimento humano denominada de Ciências Sociais.

Também é possível depreender que essas três teorias: Redes, Desenvolvimento Regional e Geografia Econômica reconhecem que os aglomerados empresariais fomentam a competitividade dos atores que neles atuam (ROHDEN; HOLLERWEGER; OSSANI, 2012; PORTER, 1992; ZAMBANINI 2014; MENDES; BELLINI, 2011; DICKEN,1986; ALLEN, 2003).

No estudo das três teorias pesquisadas foi possível identificar a transversalidade existente nas relações de trocas entre os atores, quer sejam de conhecimentos, informações ou outras, portanto, as trocas estão presentes nos relacionamentos entre eles (BALESTRIN; VARGAS 2004; ROHDEN; HOLLERWEGER; OSSANI, 2012; OLIVEIRA, 2011; MENDES; BELLINI, 2011; DICKEN,1986; ALLEN, 2003).

Por fim, (CHUNG YEUNG, 2014; BANDEIRA, 1999; OLIVEIRA, 2011; CLARK et al., 2000; SHEPPARD; BARNES, 2000; BARNES et al., 2003), concordam que as teorias de Redes, Desenvolvimento Regional e Geografia Econômica preconizam a visão de espaço geográfico e reconhecem quão positiva é a visão de espaço geográfico e a atuação regional.

\section{CONCLUSÃO}

A pesquisa utilizou a revisão sistemática no sentido de situar os conceitos de análise de rede, desenvolvimento regional e geografia econômica e organizar os fundamentos teóricos a partir de revisão da literatura em trabalhos relacionados ao tema, com isso, pesquisar se ocorre intersecção entre as teorias alvo da pesquisa.

Foi possível identificar que as três áreas do conhecimento que foram contempladas nesta pesquisa, possuem intersecção de ideias e conhecimentos em relação aos seguintes macros temas: ciências sociais; fomento à competitividade; relacionamento entre atores; visão de atuação em espaços geográficos.

As três áreas do conhecimento estudadas pertencem à macro área das Ciências Sociais, por envolverem em seus objetos, o homem e as entidades econômicas, além dos estudos de seus relacionamentos.

Entendem, também, que a atuação em formato de redes ou em aglomerados empresariais fomenta a competitividade dos atores e, para que isso ocorra, é necessário que os mesmos estejam em constantes interações como as trocas de conhecimentos e informações. Por fim, as teorias reconhecem a atuação dos agentes econômicos em mesmos espaços geográficos, permite ratificar o que antes foi encontrado, ou seja, relações de trocas e fomento à competitividade dos atores.

Sugere-se novas pesquisas em arranjos empresariais, na busca de, empiricamente, confirmar a existência da intersecção nas relações entre atores dessas redes de negócios, já que o estudo teórico ratifica essa existência.

\section{REFERÊNCIAS}

ALDRICH, D.; FELDMAN, M. R\&D spillovers and the geography of innovation and production. Americam Economic Review, v.86, n.3, p.630-640, 1996.

ALLEN, J. Lost geographies of power. Oxford: Blackwell, 2003. 
AMIN, A.; THRIFT, N. Globalisation, institutions and regional development in Europe. Oxford: Oxford

University Press, 1994, p.1-22.

BALESTRIN, A.; VARGAS, L.M. A dimensão estratégica das redes horizontais de PMEs: teorizações e evidências. Revista de Administração e Contabilidade, Curitiba, v.8, p.203-227, 2004.

BANDEIRA, P. Participação, articulação de atores sociais no desenvolvimento regional. Brasília: Instituto de Pesquisa Aplicada - IPEA, 1999.

BARNES, T.J. et al. (Ed.). Reading economic geography. Oxford. Blackwell, 2003.

BATHELT, H.; GLÜCKLER, J. Toward a relational economic geography. Journal of Economic Geography, v.3, n.2, p.117-144, 2003.

BAZZOLI, L.; DUTRAIVE, V. L'économie de l'action colletive de John Roger Commons. L'Économie Institutionnaliste: les fondateurs. V. Dutraive (Coord.). Paris, 1995. p.29-45.

BOWERSOX, D.J. The Strategic Benefits of Logistics Alliances. Harvard Business Review, Cambridge, EUA, v.68, n.4, p.36-45, 1990.

CASTRO, M.; BULGACOV, S.; HOFFMANN, V. E. Relacionamentos interorganizacionais e resultados: estudo em uma rede de cooperação horizontal da região central do Paraná. Revista de Administração e Contabilidade, Santa Catarina. v.15, n.1, p.25-46, 2011.

CHUNG YEUNG, H. W. Perspectivas das relações interorganizacionais na geografia econômica. Handbook de Relações Interorganizacionais da Oxford. Porto Alegre: Bookman, 2014, 706p.

CLARK, G.L.; FELDMAN, M.A.; GERTLER, M.S. Economic Geography; Transition and growth. The
Oxford Handbook of Economic Geography. Oxford: Oxford University Press, 2000 v.3-7.

COASE, R.H. The Nature of the Firm. Economica, New Series, v.4, n.16, p.386-405, 1937.

COX, K.R. Spaces of globalization: Reasserting the power of the local: New York: Guilford, 1997.

CUNHA, J.A.; PASSADOR, J.L.; PASSADOR, C.S. Recomendações e apontamentos para categorizações em pesquisas sobre redes interorganizacionais. Cadernos EBAPE/BR, Rio de Janeiro, v.9, Edição Especial, p.505-529, 2011.

DICKEN, P. Geographers and globalization: (Yet) another missed Boat? Transactions of the institute of British geographers, v.29q1, p.5-26, 2004.

DOMINGUES, R.A. Estratégia de crescimento? 0 caso dos royalties do setor elétrico. XVIII Encontro de Estudos Populacionais ABEP (Paper Presented). Available at: <www.abep.nepo.unicamp.br/xvii/ anais>. Accessed in: out. 2015.

FERREIRA et al. Redes organizacionais no varejo alimentar. XI SEMEAD. Anais... São Paulo: Faculdade de Economia, Administração e Contabilidade da Universidade de São Paulo, 2008.

FLEURY, A.; FLEURY, M.T.L. Internacionalização das Empresas Brasileiras: em busca de uma abordagem teórica para os late movers. In: FLEURY, A.; FLEURY, M. T.L. Internacionalização e os Países Emergentes. São Paulo: Atlas, 2010.

GIL, A.C.; OLIVA, E. de C.; SILVA, E.C. da. Desenvolvimento da regionalidade: novo campo da Administração. Regionalidade e Organizações. Programa de Pós-graduação em Administração da Universidade Municipal de São Caetano do Sul. São Paulo: Páginas e Letras, 2012a. p.11-28. 
GIL, A.C; GARCIA, C.C.; KLINK, J. Região, regionalismo e regionalidade. Regionalidade Organizações. São Paulo: Páginas \& Letras, 2012b. p.29-47.

GRABHER, G. Trading routes, bypasses, na risk intersections: mapping the travels of "network" between economic sociology and economic geography. Progresso in Human Geography, v.30, n.2, p.163-189, 2006.

GREEN, M.B. A geography of institutional stock ownership in the United States. Annals of the Association of American Geographers, v.83, n.1, p.66-89, 1993.

GUERREIRO, R.; BIO, S.R.; MENDEL, S.F. Logística integrada, gestão da cadeia de suprimentos e mensuração de custos e resultados logísticos: Um estudo com empresas brasileiras. Advances in Scientific and Applied Accounting, São Paulo, v.4, n.1, p.73-100, 2011.

HOLLERWEGER, C.; ROHDEN, S.F. Dificuldades do modelo de coopetição: um caso internacional de relações interorganizacionais. In: VII Encontro de estudos organizacionais da ANPAD, Curitiba/Paraná, Anais... Curitiba/Paraná: Anpad, 2012.

\section{JUNQUEIRA FILHO, F.O.D. A influência da} regionalidade como fator de desenvolvimento de micro e pequenas empresas: Um estudo do APL vinícula de Jundiaí - SP. 2006. 105f. Dissertação (Mestrado em Administração) - Programa de PósGraduação em Administração da Universidade Municipal de São Caetano do Sul, São Caetano do Sul-São Paulo, 2006.

KALMANOVITZ, S. El neo institucionalismo como escuela. Revista de Economía Institucional, Colombia, v.5, n.9, p.189-215, 2003.
KELLY, P.F. The geographies and politics of globalization. Progress in Human Geography, v.23, n.3, p.379-400, 1999

LUKES, S. Power. Oxford: Blackwell, 1996.

LUO, Y. Toward coopetition within a multinational enterprise: a perspective from foreign subsidiarie. Journal of World Business, Pennsylvania, USA v.40, p.71-90, 2005.

MALMBERG, A.; MASKELL, P. Toward an explanation of regional specialization and industry agglomeration. European Planning Studies. V.5, p.25-41, 1997.

MARTIN, R. Geografia humana - sociedade, espaço e ciência social. São Paulo-SP: Jorge Zahar, 1996.

MASKELL, P.; MALMBERG, A. Localised learning and industrial competitiveness. Cambridge Journal of Economics. v.23, n.2, p.167-185, 1999.

MEHIER, C. Les apports de Clarence Edwin Ayres: place et rôle de la tecnologie dans la dynamique économique. In : DUTRAIVE, V. (Coord.). L’Économie Institutionaliste: les fondateurs. Paris: Economica, 1995. p.61-75.

MENDES, M.R.; BELLINI, M.I.B. Textos e contextos. Porto Alegre: EDIPUCRS, 2011.

NALEBUFF, B.J; BRANDENBURGER, A.M. Coopetição. Rio de Janeiro: Rocco, 1996.

NORTH, D.C. Desempeño económico en el transcurso de los años. In: Conferencia de North em Estocolmo, Suecia, el 09 diciembre, al recibir el Premio Nobel de Ciencias Económicas. 26p. Available at: <http:// www.eumed.net>. Accessed in: 3 maio 2016. 
OLDS, K. et al. Globalisation and the Asia-Pacific: Contested Territories. London: Routledge, 1999.

\section{OLIVEIRA, I.S.V. Relacionamentos} interorganizacionais como fonte de vantagem competitiva: um estudo em empresas do APL de calçados da grande João Pessoa-PB. 2014. 203f. Dissertação (Mestrado em Engenharia de Produção) - Universidade Federal da Paraíba, João Pessoa-PB, 2014.

PATTON, M.Q. How to use qualitative methods in evaluation. Newbury Park, CA: Sage, 1987.

PECK, J.A.; YEUNG, H.W. Remaking the global economy: Economic-Geographical perspectives. London: Sage, 2005.

PFEFFER, J.; NOWAK, P. Joint ventures and interorganizational interdependence. Administrative Science Quarterly, v.21, p.398-418, 1976.

PHELPS, N.A. Clusters, dispersion and the spaces in between: For an economic geography of the banal. Urban Studies. v.41, n.5-6, p.971-989, 2004.

PITTERI, S.; SAES, M.S.M.; BRESCIANI, L.P. Competências territoriais e desenvolvimento regional: Uma proposta teórico-metodológica para pesquisas interdisciplinares. Desenvolvimento em Questão, Unijuí, n.31, jul-set. 2015.

PORTER, M.E. Estratégia competitiva: técnicas para análise de indústria e da concorrência. 7.ed. Rio de Janeiro: Campus, 1986.

PORTER, M. E. Vantagem competitiva. Rio de Janeiro: Campos 1992.
ROHDEN, S.F.; HOLLERWEGER, C.; OSSANI, A. Benefícios em Redes de cooperação: a perspectiva da rede das redes. In: VII Encontro dos estudos organizacionais da ANPAD, Curitiba/Paraná, Anais... Curitiba-Paraná: ANPAD, maio 2012.

SAMPAIO, R.F.; MANCINI, M.C. Estudos de revisão sistemática: um guia para síntese criteriosa da evidência científica. Revista Brasileira de Fisioterapia, São Carlos, v.11, n.1, p.83-89, jan-fev.2007.

SCOTT, A.J. New industrial space: flexible production, organization and regional development in north America and western Europe. London: Pion, 1988.

SCOTT, A.J. Regions and the World Economy: the coming shap of global productions, competition and political order. Oxford: Oxford University Press, 1998.

SHEPPARD, E.; BARNES, T.J. A companion to economic geography. Oxford: The Handbook of Economic Sociology, $2^{\text {nd }}$ edn. Priceton University Press, 2000

STORPER, M. The regional wolrd: Territorial development in a global economy. New York: Guilford Press, 1997.

\section{WILLIAMSON, O.E. Transactions cost economics} and organization theory. Berkeley: University of California, 1993. 58p.

YEUNG.H.C. The firm as social network: An organizational perspective. Growth and change. Progress in Human Geograhy, v.36, n.3, p.307-328, 2005.

YEUNG.H.C. Capital, State and Space: Contesting the Borderless World. Transactions of the Institute of British Geographers, v.23, n.3, p.291-309, 1998. 
ZAMBANINI, M.E. Inovação e desenvolvimento local: uma análise sobre São José dos Campos. 2014. $196 f$. Tese (Doutorado em Administração) - Universidade Municipal de São Caetano do Sul, São Caetano do Sul-São Paulo, 2014
ZINELDIN, M. Coopetition: the organization of the future. Marketing Intelligence \& Planning, v.22, p.780-789, 2004.

1 Aluna de doutorado em Administração do Programa PPAD da Universidade da Amazônia; Contadora e mestre em Administração. E-mail: barbaraadria@yahoo.com.br

2 Agrônoma e Mestre em Extensão Rural - Universidade Federal de Viçosa; Professora do PPAD da Universidade da Amazônia; Doutora em Ciências Agrárias. E-mail: cyntiamei@hotmail.com Universidade da Amazônia; Mestre em Controladoria - FEA/USP; 